\title{
Light scattering resonances in small particles with electric and magnetic properties
}

\author{
B. García-Cámara, ${ }^{1, *}$ F. Moreno, ${ }^{1}$ F. González, ${ }^{1}$ J. M. Saiz, ${ }^{1}$ and G. Videen ${ }^{2}$ \\ ${ }^{1}$ Grupo de Óptica, Departamento de Física Aplicada, Universidad de Cantabria, Avda. de los Castros s/n, 39005 , \\ Santander, Spain \\ ${ }^{2}$ Army Research Laboratory, 2800 Powder Mill Road, Adelphi, Maryland 20783, USA \\ *Corresponding author: garciacb@unican.es
}

Received August 16, 2007; revised November 21, 2007; accepted November 21, 2007; posted December 4, 2007 (Doc. ID 86559); published January 10, 2008

\begin{abstract}
Lorenz-Mie resonances produced by small spheres are analyzed as a function of their size and optical properties $(\varepsilon \gtrless 0, \mu \gtrless 0)$. New generalized $(\mu \neq 1)$ approximate and compact expressions of the first four Lorenz-Mie coefficients $\left(a_{1}, b_{1}, a_{2}\right.$, and $\left.b_{2}\right)$ are calculated. With these expressions and for small particles with various values of $\varepsilon$ and $\mu$, the extinction cross section $\left(Q_{e x t}\right)$ is calculated and analyzed, in particular for resonant conditions. The dependence on particle size of the extinction resonance, together with the resonance shape (FWHM), is also analyzed. In addition to the former analysis, a study of the scattering diagrams for some interesting values of $\varepsilon$ and $\mu$ is also presented. (C) 2008 Optical Society of America

OCIS codes: $290.2200,290.4020,290.5850,350.3618$.
\end{abstract}

\section{INTRODUCTION}

The problem of electromagnetic scattering from a homogeneous sphere of arbitrary size was solved by Lorenz and Mie many years ago [1,2]. The Lorenz-Mie theory gives the scattering properties of a sphere immersed in a homogeneous and isotropic medium, provided that the sphere material is also isotropic and is characterized by a dielectric constant, which can be complex and frequency dependent. By means of this solution, both the absorption and scattering cross sections are obtained as a function of the well known Lorenz-Mie scattering coefficients [3,4].

The study of light scattering from particles of size much smaller than the incident wavelength (Rayleigh scatterers) has become an attractive topic in the last years because of the interest that has arisen especially in the field of nanotechnology. The possibility of exciting resonances in metallic nanoparticles, and consequently, the generation of intense local electric fields [localized surface plasmons (LSP)], has found important applications in the biomedical sciences (biosensors, nanorulers, molecular orientation sensing, etc.) and industry (enhancement of photodetection in detectors and solar cells, light guiding in telecommunications, optical computing, etc.) [5-9]. Early studies of these resonances were made by Ruppin in the 1970's [10,11], but it is relatively recent that numerous works regarding such resonances from metallic isolated nanoparticles, dimers, aggregates of nanoparticles, or nanoparticles on surfaces have been developed either with a theoretical or applied scope [12-19].

The Rayleigh scattering approximation requires two conditions. First, the size parameter $x(x=2 \pi R / \lambda$, where $R$ is the radius of the sphere and $\lambda$ is the illuminating wavelength) should be much smaller than one, which is the limiting case of the Lorenz-Mie formulation. Second, the refractive index $m$ also must be small, in such a way that $|m| x \ll 1$. Under these conditions, scattering of elec- tromagnetic radiation is dominated by the first electric term of the Lorenz-Mie series expansion. Videen and Bickel [20] examined what happens when the second condition is relaxed for very small, nonmagnetic $(\mu=1)$ spheres and found the existence of new resonances for such particles. They further derived simplified expressions for the scattering coefficients that could be used to examine the behavior of these resonances as a function of system parameters like the refractive index and radius of the sphere.

For the case in which the isolated particles show a magnetic response to the incident field $(\mu \neq 1)$ and their size is much smaller than the incident wavelength, the scattering properties have received much less attention. These particles have interesting scattering properties [21-23]. Also, with the recent appearance of engineered materials (known as "metamaterials") whose optical properties can be controlled, light scattering by small particles with exceptional optical properties (for instance $\varepsilon<0$ and $\mu<0)$ is receiving a lot of attention from the scientific community interested in this field [24-30]. We cite, as an example, the possibility of building optical nanocircuits based on metamaterials for optical communication and computing applications [31].

With this in mind, the objective of our research is to generalize that performed by Videen and Bickel [20] for the case of small particles with no restriction on the values of $\varepsilon$ and $\mu$. We present analytical expressions of the first four Mie coefficients $\left(a_{1}, a_{2}, b_{1}, b_{2}\right)$ that allow us to calculate and to examine the resonance behavior of the particles as a function of their size and optical properties. Special attention will be paid to the case $\varepsilon<0$ and $\mu<0$.

The work is organized as follows. Section 2 is devoted to presenting generalized and simplified expressions of the Lorenz-Mie coefficients $a_{1}, a_{2}, b_{1}$, and $b_{2}$. In Section 3 , we analyze the Lorenz-Mie resonances as a function of 
the optical properties and the size of the particles. In Section 4 , we show and analyze the scattering diagrams of the particles for different situations. Finally, the last section summarizes the main conclusions.

\section{SCATTERING COEFFICIENTS}

For a homogeneous and isotropic sphere of radius $R$ illuminated by a plane wave of angular frequency $\omega$ traveling in the $z$ direction $[\vec{k}=(0,0, k)=(0,0, \omega / c), k$ being the wavenumber in the surrounding medium] and linearly polarized in the $x$ direction, the scattered electromagnetic field can be calculated in every point of space $\vec{r}$ according to the Lorenz-Mie theory [3] as

$$
\begin{gathered}
\mathbf{E}_{s}(\vec{r})=\sum_{n=1}^{\infty} E_{n}\left[i a_{n} \mathbf{N}_{e l n}^{(3)}(k \vec{r})-b_{n} \mathbf{M}_{o l n}^{(3)}(k \vec{r})\right], \\
\mathbf{H}_{s}(\vec{r})=\sqrt{\frac{\varepsilon}{\mu} \sum_{n=1}^{\infty} E_{n}\left[i b_{n} \mathbf{N}_{o l n}^{(3)}(k \vec{r})-a_{n} \mathbf{M}_{e l n}^{(3)}(k \vec{r})\right] .}
\end{gathered}
$$

In Eq. (1), $a_{n}$ and $b_{n}$ are the Lorenz-Mie coefficients; $\mathbf{N}_{e l n}$, $\mathbf{M}_{o l n}, \mathbf{N}_{o l n}$, and $\mathbf{M}_{\text {eln }}$ are the vector spherical harmonics; $E_{n}=i^{n} E_{0}(2 n+1) / n(n+1)$, with $E_{0}$ being the amplitude of the incident electric field, and $\varepsilon, \mu$ are the relative (to the vacuum) electric permittivity and the relative (to the vacuum) magnetic permeability of the sphere, respectively.

The general expressions for the Mie coefficients, including the magnetic contribution, are given by

$$
\begin{aligned}
a_{n} & =\frac{\tilde{m} \psi_{n}^{\prime}(x) \psi_{n}(m x)-\psi_{n}^{\prime}(m x) \psi_{n}(x)}{\tilde{m} \xi_{n}^{\prime}(x) \psi_{n}(m x)-\psi_{n}^{\prime}(m x) \xi_{n}(x)}, \\
b_{n} & =\frac{\tilde{m} \psi_{n}^{\prime}(x) \psi_{n}(m x)-\psi_{n}^{\prime}(m x) \psi_{n}(x)}{\tilde{m} \psi_{n}^{\prime}(x) \xi_{n}(m x)-\xi_{n}^{\prime}(m x) \psi_{n}(x)},
\end{aligned}
$$

where $x$ is the size parameter, $m=\sqrt{\varepsilon \mu}$ is the complex refractive index of the sphere, $\tilde{m}=m / \mu$, and $\psi_{n}, \xi_{n}$ are the spherical Ricatti-Bessel functions.
The extinction efficiency can be expressed as a sum of these coefficients as follows

$$
Q_{\text {ext }}=\frac{2}{x^{2}} \sum_{n}(2 n+1) \operatorname{Re}\left(a_{n}+b_{n}\right) .
$$

In the Rayleigh limit, $x \ll 1$ and $|m| x \ll 1$, only the firstorder coefficients contribute to the scattered field and they can be approximated by [3]

$$
\begin{gathered}
a_{1} \approx \frac{2 i}{3} x^{3} \frac{\varepsilon-1}{\varepsilon+2}, \quad b_{1} \approx \frac{2 i}{3} x^{3} \frac{\mu-1}{\mu+2} \\
a_{n} \approx b_{n} \approx 0 \text { for } n>1
\end{gathered}
$$

That is, only the dipolar contribution is important when the particle size is smaller than the incident wavelength, and quadrupolar and higher-order terms can be taken as zero. As can be seen in relations (4), $a_{1}$ includes only the relative electric permittivity $\varepsilon$, and $b_{1}$ includes only the relative magnetic permeability $\mu$. For this reason we may refer to $a_{n}$ or $b_{n}$ terms as electric or magnetic terms, respectively, and the same for their corresponding resonances.

When the condition $x \ll 1$ is satisfied but not the condition $|m| x \ll 1$, the approximations leading to relations (4) are no longer valid. In this case, Videen and Bickel [20] derived expressions for $a_{1}$ and $a_{2}$ (and because of the symmetry, also for $b_{1}$ and $b_{2}$ ) that are accurate for dielectric and nonmagnetic spheres $(\varepsilon<0$ and $\mu=1)$. Here, we address the general case $(\varepsilon, \mu \neq 1)$ as follows: From the condition of $x \ll 1$, we substituted sine and cosine functions whose argument is the size parameter $x$ by the first two terms of their Taylor expansion and neglected the contribution of higher-order powers. The resulting coefficients are approximate expressions valid for any pair of optical constants $(\varepsilon, \mu)$ and can be written as

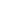

$$
a_{1}=\frac{\tilde{m} m x^{3}[m x \cdot \cos (m x)-\sin (m x)]}{\cos (m x)\left(-\tilde{m} m^{2} x^{4}-i \tilde{m} m^{2} x+i m x^{3}+i m x\right)+\sin (m x)\left(\tilde{m} m x^{3}+i \tilde{m} m-i x^{2}+i m^{2} x^{4}-i+i m^{2} x^{2}\right)},
$$

$\cos (m x)\left(6 \tilde{m} m^{2} x^{2}-6 m x^{2}+m^{3} x^{4}\right)+\sin (m x)\left(6 x-3 m^{2} x^{3}-6 \tilde{m} m x+2 \tilde{m} m^{3} x^{3}\right)$

$a_{2}=\frac{\left\{\cos (m x)\left[3 i \tilde{m} m^{3} x^{2}+2 \tilde{m} m^{3} x^{3}-i \tilde{m} m^{3} x^{4}+6 i \tilde{m} m^{3}-9 i \tilde{m} m-6 \tilde{m} m x+3 i \tilde{m} m x^{2}+18 \frac{i \tilde{m} m}{x^{2}}-\left(-\frac{3 i}{x^{2}}-2 i-x\right)\left(6-3 m^{2} x^{2}\right)\right]+\sin (m x)\left[9 i \tilde{m} m^{2} x+6 \tilde{m} m^{2} x^{2}-3 i \tilde{m} m^{2} x^{3}+18 \frac{i \tilde{m} m^{2}}{x}-\left(-\frac{3 i}{x^{2}}-2 i-x\right)\left(-6 m x+m^{3} x^{3}\right)\right]\right\}}{x}$

Because of the symmetry of the scattering coefficients [20]

$$
b_{n}(1 / \tilde{m}, m, x)=a_{n}(\tilde{m}, m, x) .
$$

In Fig. 1 we show $\log \left(Q_{\text {ext }}\right)$ obtained for an isolated particle with $R=0.01 \lambda$ for the cases $\varepsilon<0, \mu=1$ [Fig. 1(a)] and $\varepsilon>0, \mu=1$ [Fig. 1(b)]. In both, we used three methods to calculate the extinction efficiency: (i) The exact Mie coefficients, (ii) our approximate coefficients (AC) given by Eqs. (5) (AC1), and (iii) other approximate expressions but with the first four terms of the Taylor expansion of the functions' sine and cosine (AC2). As can be seen, our ex- 


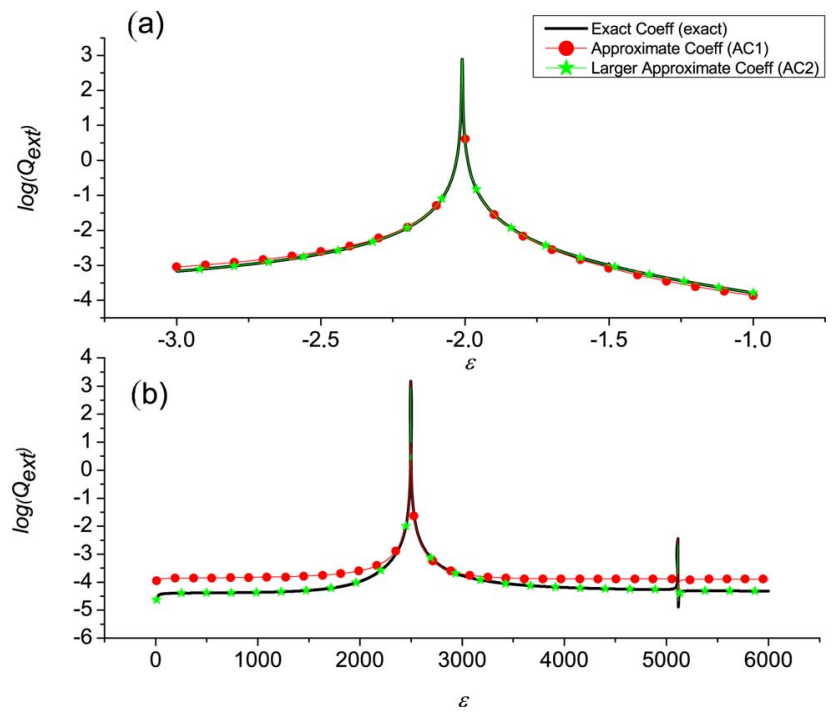

Fig. 1. (Color online) Comparative plot of $Q_{\text {ext }}$ for three different expressions of Mie coefficients: Exact (solid curve), approximate with Eqs. (5) and (6) (AC1), and approximate using more coefficients in the expansion of sine and cosine (AC2) for $\mu=1$. (a) Metallic case $(\varepsilon<0)$, (b) dielectric case $(\varepsilon>0)$.

pressions reproduce very accurately the position of the resonances and allow us to make a qualitative study of the width of them.

It can be seen in Fig. 1(b) that for the dielectric case and outside the resonance, the values of $\log \left(Q_{\text {ext }}\right)$ calculated with the reduced coefficients proposed here differ from exact values. To reproduce this area exactly, a longer expansion of trigonometric functions is needed. However, this gives more complex expressions for the coefficients $a_{n}$ and $b_{n}$ than those of Eqs. (5) and (6). In what follows, we will use these equations, as the main interest of this research is focused on the analysis of resonances, in particular their shape (FWHM) and position.

(a)

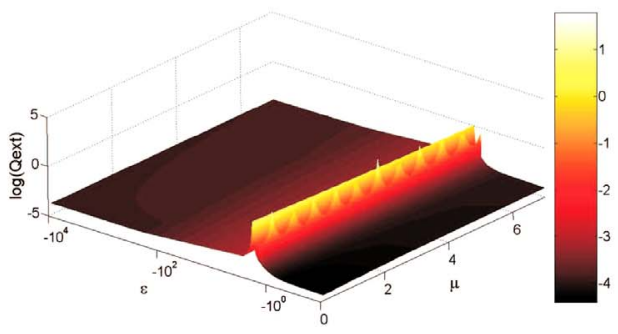

(c)

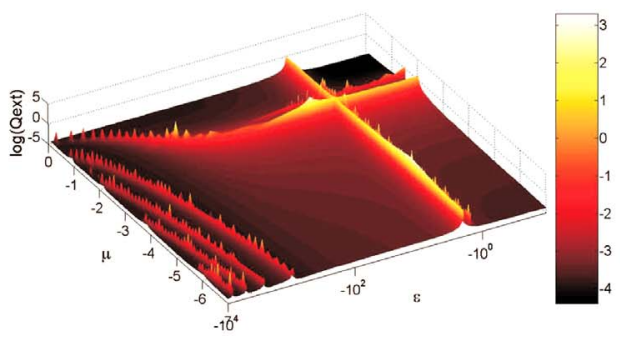

\section{RESONANCES}

Resonant scattering in a spherical particle is caused by a charge oscillation resonance inside the particle, and is therefore determined by the optical properties at the incident frequency and the size of the particle, i.e., the constituents of the resonator. From a mathematical point of view and for small particles, resonances appear when the denominators of the Lorenz-Mie coefficients [Eq. (2)] are equal to zero. In this section, we analyze the dependence of these zeros on both the optical properties of the particle and its size.

For small particle size, we consider only values that allow us to use the first four Lorenz-Mie coefficients, so that Eq. (3) can be written as

$$
Q_{e x t}=\frac{2}{x^{2}}\left[3 \operatorname{Re}\left(a_{1}+b_{1}\right)+5 \operatorname{Re}\left(a_{2}+b_{2}\right)\right] .
$$

In this equation we use the approximate expressions for $a_{1}, a_{2}, b_{1}$, and $b_{2}$ shown in Eq. (5), where $a_{1}$ and $b_{1}$ are the dipolar terms (electric and magnetic, respectively), and $a_{2}, b_{2}$ are the quadrupolar ones. Fig. 2 shows a 3D plot of $\log \left(Q_{\text {ext }}\right)$ as a function of $(\varepsilon, \mu)$ for a sphere of radius $R$ $=0.01 \lambda$, including every possibility $(\varepsilon \gtrless 0$ and $\mu \gtrless 0)$. Note that semilog representation is used. Also, the symmetry between coefficients $a_{n}$ and $b_{n}$ given by Eq. (6) has been considered. Some interesting features in the resonance regions of this plot have been enlarged and are shown in Fig. 3 for clarity.

Case $\epsilon>0, \mu>0$ : First, when $\epsilon>0, \mu=1$ the well-known Lorenz-Mie resonances appear. Resonances are organized in branches, each having an origin in the electric or magnetic terms depending on the values taken by $\epsilon$ and $\mu$. By increasing the magnetic permeability, resonances shift to smaller values of $\epsilon$ in a discontinuous sequence as can be seen in Fig. 3(a). The coefficient associated with a given branch of resonances is indicated in the figure.

(b)

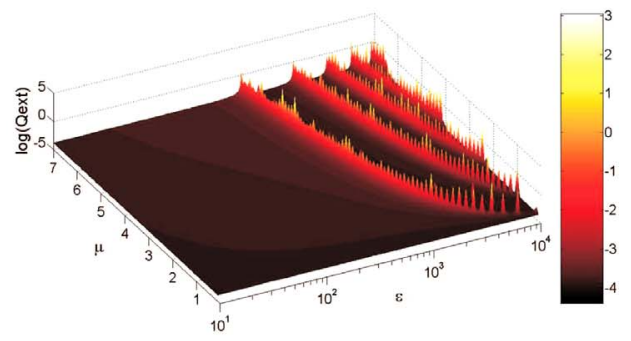

(d)

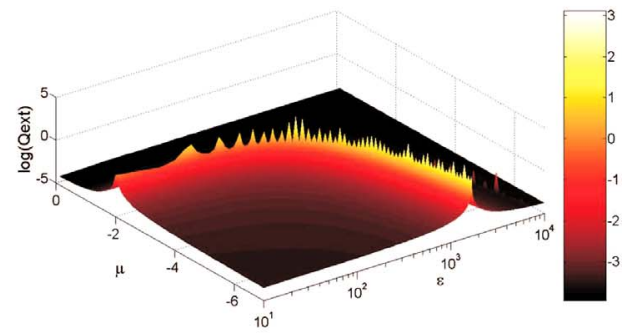

Fig. 2. (Color online) 3D plots of $\log \left(Q_{\text {ext }}\right)$ as a function of the optical properties $(\varepsilon$ and $\mu$ ) for a spherical particle of $R=0.01 \lambda$. 
(a)

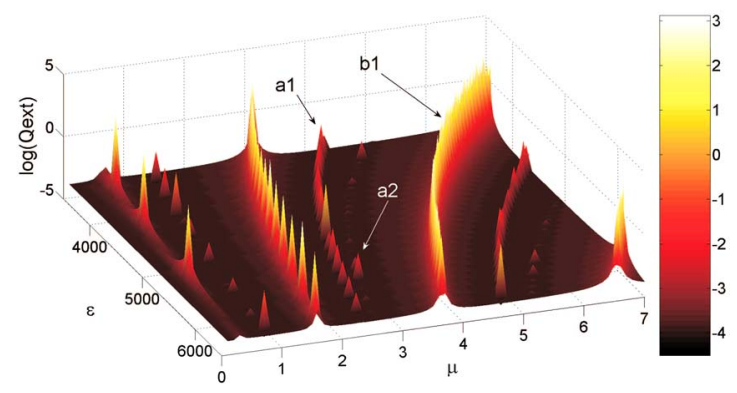

(b)

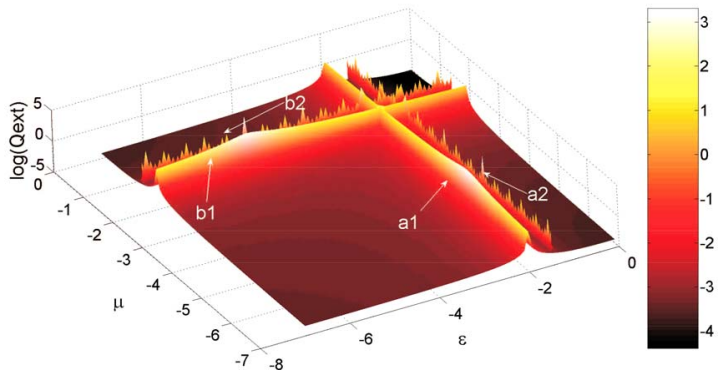

Fig. 3. (Color online) Enlargement of two interesting zones of Fig. 2: (a) Region with $\varepsilon>0, \mu>1$; (b) region with $\varepsilon<0, \mu<0$. The coefficient that takes the highest value when this resonance is excited is indicated.

Case $\epsilon<0, \mu>0$ : Only electric resonances are observed in this region ( $a_{1}$ or $a_{2}$ may take high values). Dipolar resonances due to high values of $a_{1}$ appear around $\epsilon \cong$ -2 , reaching the exact value when the particle size approaches zero. Electric quadrupolar resonances $\left(a_{2}\right)$ appear around $\epsilon \cong-1.5$.

Case $\epsilon<0, \mu<0$ : This region corresponds to the wellknown anomalous situation of a negative refractive index [24]. In this region and for low values of the modulus of the electric permittivity $(|\epsilon| \leqslant 10)$, both magnetic and electric resonances are observed [Fig. 3(b)]. The electric ones appear for the same values of $\epsilon$ as those observed for $\mu$ $>0$ in the previous case: $\epsilon \cong-2, \epsilon \cong-1.5$. Another two resonances appear associated with coefficients $b_{1}$ and $b_{2}$. The dipolar one (corresponding to coefficient $b_{1}$ ) is located around $\mu \cong-2$, and the quadrupolar (corresponding to coefficient $b_{2}$ ) at $\mu \cong-1.5$. As we can see, there is an interesting $\epsilon-\mu$ symmetry. The electric modes appear for values of $\epsilon$ equal to those of $\mu$ for which the magnetic modes appear. This symmetry is similar to that observed in Mie coefficients for dipolar modes and in the very small particle regime [Eq. (4)] but now extended to the quadrupolar modes.

For larger values of the electric permittivity $\left(|\epsilon|>10^{2}\right)$ the behavior of the resonances is similar to that found for $\epsilon>0$ and $\mu>0$, in the sense that by increasing the modulus of the magnetic permeability $\mu$ resonances shift to lower values of $|\epsilon|$.

Case $\epsilon>0, \mu<0$ : For $\epsilon>0$ and $\mu<0$ only the magnetic dipolar or quadrupolar modes (coefficients $b_{1}$ and $b_{2}$, respectively) appear. For a large range of values of $\epsilon$, whose length depends on the actual radius of the particle, the magnetic modes are located around $\mu \cong-2$ and $\mu \cong-1.5$ for dipolar and quadrupolar modes, respectively. Out of this range the location of the resonances has a more com- plex behavior. In Fig. 4 two different regions are shown: $\epsilon<0, \mu>0$ [Fig. 4(a)] and $\epsilon>0, \mu<0$ [Fig. 4(b)]. As can be seen, in these ranges there is $\epsilon-\mu$ symmetry, similar to that described above.

Another interesting feature observed in these plots is the resonant range for either the $\epsilon$ or $\mu$ constant. These resonant intervals and their actual shape are very sensitive to the particle size.

The simplicity of the expressions found for coefficients $a_{1}, a_{2}, b_{1}$, and $b_{2}$ make it very easy to extend our former analysis to other values of the particle radius. As an example, Fig. 5 shows, for $\epsilon<0, \mu<0$, the curves obtained when $\log \left(Q_{\text {ext }}\right)$ is plotted against $\epsilon$ for a resonant $\mu$ and for several values of the particle radius. Resonant permeability values $\mu=-2$ and $\mu=-1.5$ correspond to Figs. 5 (a) and 5(b), respectively. Again the coefficient that takes the highest value in a maximum is labeled in the figure.

As can be observed in Fig. 5(a) only dipolar resonances are observed in the case $\mu=-2$ for very small particles $(R=0.01 \lambda)$ : the electric one due to $a_{1}$ and located at $\epsilon \cong$ -2 , and the magnetic one due to $b_{1}$ and located at $\mu \sim$ -5 . As the particle size increases, the parameter $Q_{\text {ext }}$ takes lower values on these resonances, and a new resonance appears at $\epsilon \cong-1.5$ due to coefficient $a_{2}$. For even higher values of the particle radius, only the electric quadrupolar resonance associated with $a_{2}$ remains as a welldefined resonance.

In the case $\mu=-1.5$ [Fig. 5(b)] the quadrupolar magnetic resonance $(\epsilon \sim-2.33)$, associated with coefficient $b_{2}$ is observed even for the lowest values of the particle size $(R=0.01 \lambda)$. As the particle size increases, the resonance

(a)
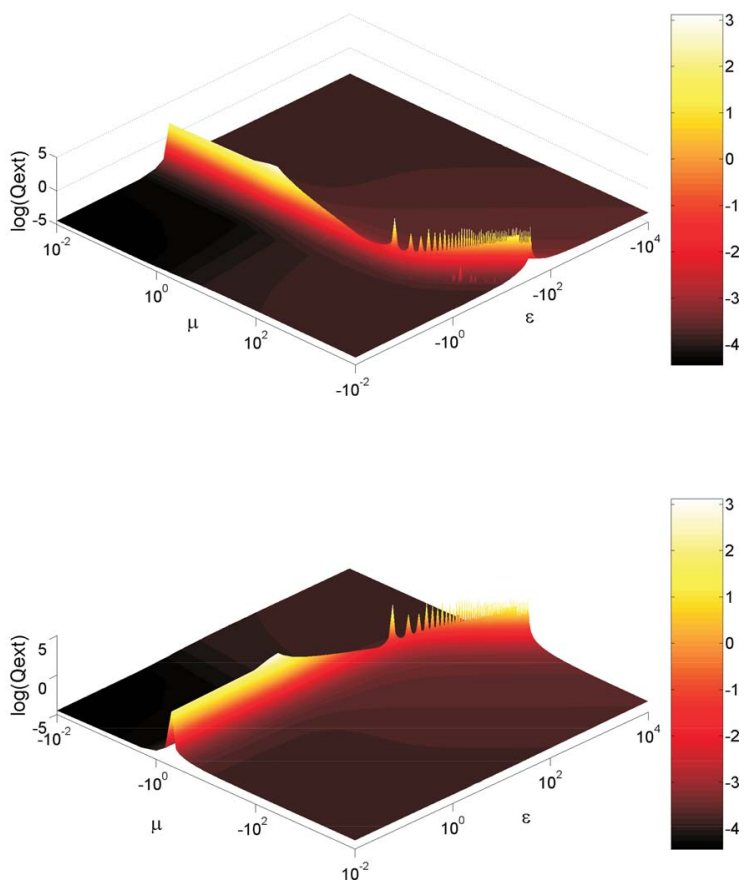

Fig. 4. (Color online) 3D plots of $\log \left(Q_{\text {ext }}\right)$ as a function of the optical properties ( $\varepsilon$ and $\mu$ ) for two different ranges: (a) $\varepsilon<0$ and $\mu>0$, (b) $\varepsilon>0$ and $\mu<0$, when the range of values of $\varepsilon$ and $\mu$ is equal. 
(a)

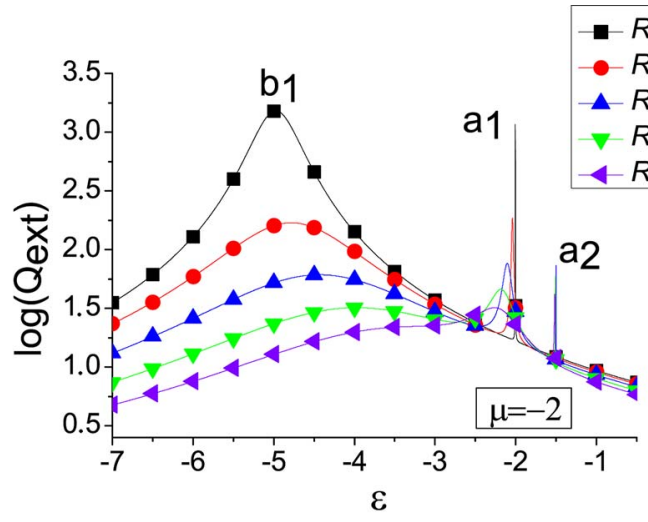

(b)

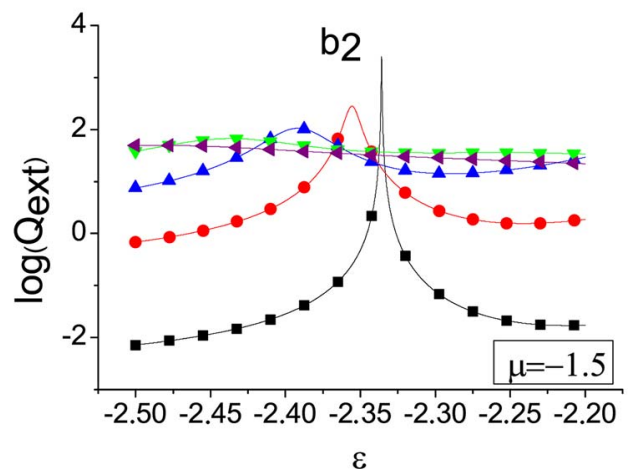

Fig. 5. (Color online) Evolution of the extinction cross section as a function of the electric permittivity in the range $\varepsilon<0$ for different values of particle radius $R$. The value of the magnetic permeability is indicated in the bottom right corner. Resonances are labeled with the Lorenz-Mie coefficient that takes the highest value at this point. shifts and broadens, finally losing its well-defined shape, while the values of $Q_{\text {ext }}$ grow within a large range of values of $\epsilon$.

As was mentioned before, the validity of Eqs. (5) and (6) and their corresponding expression for $b_{i}$ over a wide range of values of $R$ and for any pair of values $(\epsilon, \mu)$ allows a systematic study of the resonances as a function of the particle size, including the two main features observed: the location and the width of such resonances. Fig. 6 shows the evolution of the location $\left(\epsilon_{\max }\right)$ and width FWHM of four different resonances, each associated with a different coefficient, as a function of the particle radius in the range $R / \lambda \in[0.01,0.1]$. In all cases $\epsilon$ is taken as negative, and the resonant value of $\mu$ is indicated in the lower part of the figures.

For the electric resonances shown in Figs. 6(a) and 6(b) the position of $\epsilon_{\max }$ shifts to higher values of $|\epsilon|$ as $R$ increases, the dipolar resonance showing a larger shift (from $\epsilon=-2$ to $\epsilon=-2.3$ ) than that of the quadrupolar one (from $\epsilon=-1.5$ to $\epsilon=-1.525$ ).

For the magnetic resonances shown in Figs. 6(c) and 6(d) the shift of $\epsilon_{\max }$ is different for coefficients $b_{1}$ and $b_{2}$. While the dipolar $b_{1}$ tends to lower values of $|\epsilon|$ when particle size increases, the quadupolar $b_{2}$ moves in the opposite direction. Again, the shift of the dipolar resonance is larger than that of the quadrupolar one.

Concerning resonance FWHM it is clear that in all situations under study for $\epsilon<0$, the smaller the particle size, the narrower the resonance peak. In other words, slopes are positive in all FWHM curves of Fig. 6, the effect being more noticeable in the case of dipolar resonances. (a)

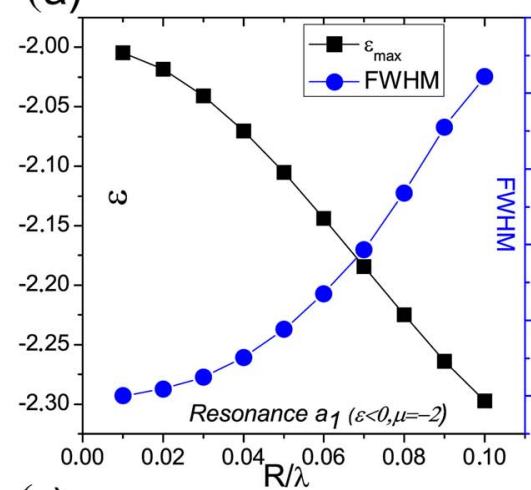

(c)

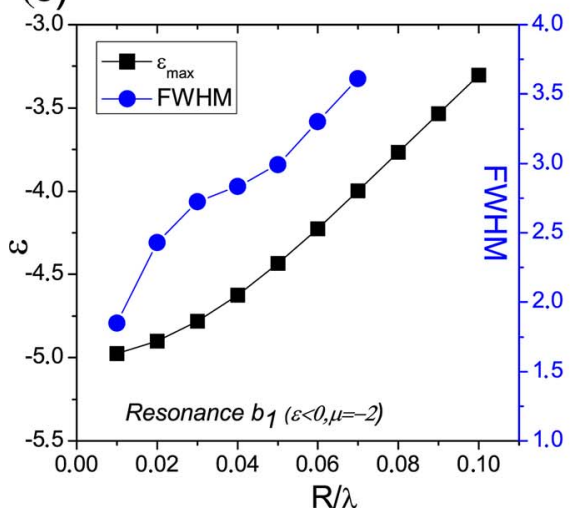

(b)

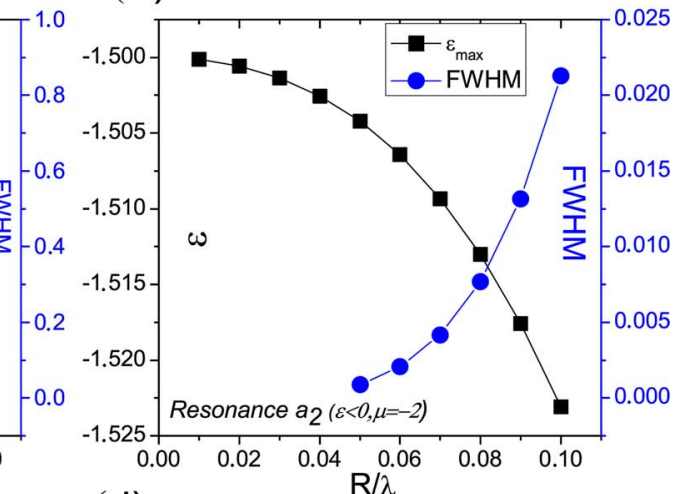

(d)

(d)

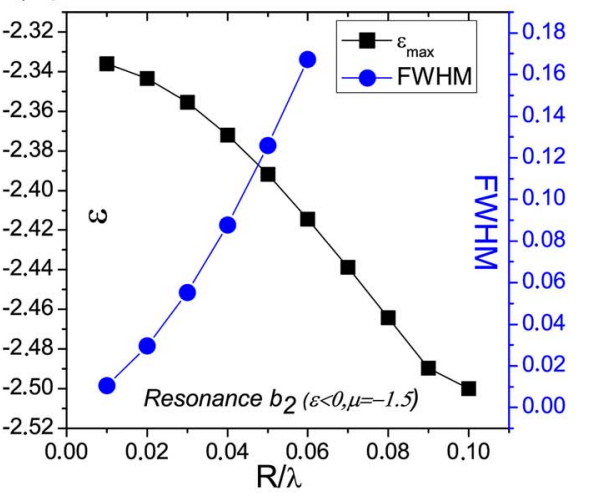

Fig. 6. (Color online) Evolution of FWHM and position of the resonances as a function of particle size. 


\section{SCATTERING DIAGRAMS}

In addition to our former analysis, we show in this section the far-field scattering results corresponding to spherical particles of different size and properties, considering the scattering patterns associated with the resonant conditions established in the previous section. Incident linearly polarized beams will be assumed, and the scattering plane will be chosen perpendicular (TE) or parallel (TM) to incident polarization.

For values of $R$ lower than $0.1 \lambda$, our approximate coefficients reproduce very accurately the scattering diagrams obtained by using the exact Mie coefficients. In Fig. 7 and Fig. 8, we show for the electric and magnetic resonances, respectively, the scattering patterns for a set of five different sizes $(0.01,0.02,0.03,0.04$, and $0.05 \lambda)$. For the results shown in Fig. 7(a) and 7(b), $\mu=-2$ and $\epsilon \approx-2$ (the exact value depends on the excitation of the dipolar resonance associated with $a_{1}$ for each particle size) where the electric dipolar resonance associated with $a_{1}$ is excited (see Fig. 5 for a better identification of these values). For the results shown in Figs. 7(c) and 7(d), $\mu=-2$ and $\epsilon \approx$ -1.5 (the exact value depends on the excitation of the quadrupolar resonance associated with $a_{2}$ for each particle size) where the electric quadrupolar resonance associated with $a_{2}$ is excited.

Figures 8(a) and 8(b) correspond to a case in which the magnetic dipolar resonance due to $b_{1}$ is excited: $\mu=-2$ and $\epsilon \sim-5$ (again with small variations depending on the particle size) while in Figs. 8(c) and 8(d) the magnetic quadrupolar resonance associated with coefficient $b_{2}$ is excited by choosing the values $\mu=-1.5$ and $\epsilon \sim-2.3$ (with corresponding small variations due to particle size).

All these figures have been normalized to the maximum value (not always the forward scattering) for better observation of the evolution of the angular structure, thus allowing no absolute comparison among patterns of different sizes, properties, or polarization. In Table 1, we include the maximum values of the scattered intensity (and its corresponding scattering angle) for each case for possible further comparative calculations.

The range of particle radii has been selected for the purpose of observing the interesting changes produced in the backscattering direction (let us remember that larger particles will produce a dominant scattering in the forward direction). For instance, the dipolar electric resonance, Figs. 7(a) and 7(b), shows an evolution from an almost null backscattering to a considerable one at $R$ $=0.05 \lambda$. The null backscattering is explained by the proximity at $\epsilon$ to -2 for the smallest particle, almost satisfying the condition $\epsilon=\mu$ [21]. This condition is less satisfied when the sizes increases, and the backscattered intensity increases. For the quadrupolar electric resonance, scattering at $90^{\circ}$ seems to be more sensitive to polarization, and whole observation in the TE case does not produce important size-dependent changes. In the TM case, an important angular dependence arises in the interval from $R=0.01 \lambda$ to $0.05 \lambda$, reaching the characteristic shape of a quadrupolar. This means that quadrupolar terms are becoming dominant for that size.

When the dipolar magnetic resonance is excited, Figs. 8(a) and Fig. 8(b), the classic shape of an emitting dipole is observed, though the shapes for TE and TM modes are inverted with respect to those of an electric dipole. As $R$ increases the scattering values also increases, but with no noticeable change in the shape.

In Figs. 8(c) and 8(d) the magnetic quadrupolar resonance appears very well defined, even for particles as small as $R=0.01 \lambda$.

As above, in Table 2 we include the maximum values of the scattered intensity (and its corresponding scattering angle) for each case for possible further comparative calculations.

A final remark about these results must be made. The combination $\mu=-2$ and $\epsilon \sim-2$ satisfies Kerker's condition [21] for obtaining zero forward scattering, but, as can be observed in Fig. 7, the resonance obtained for this par-
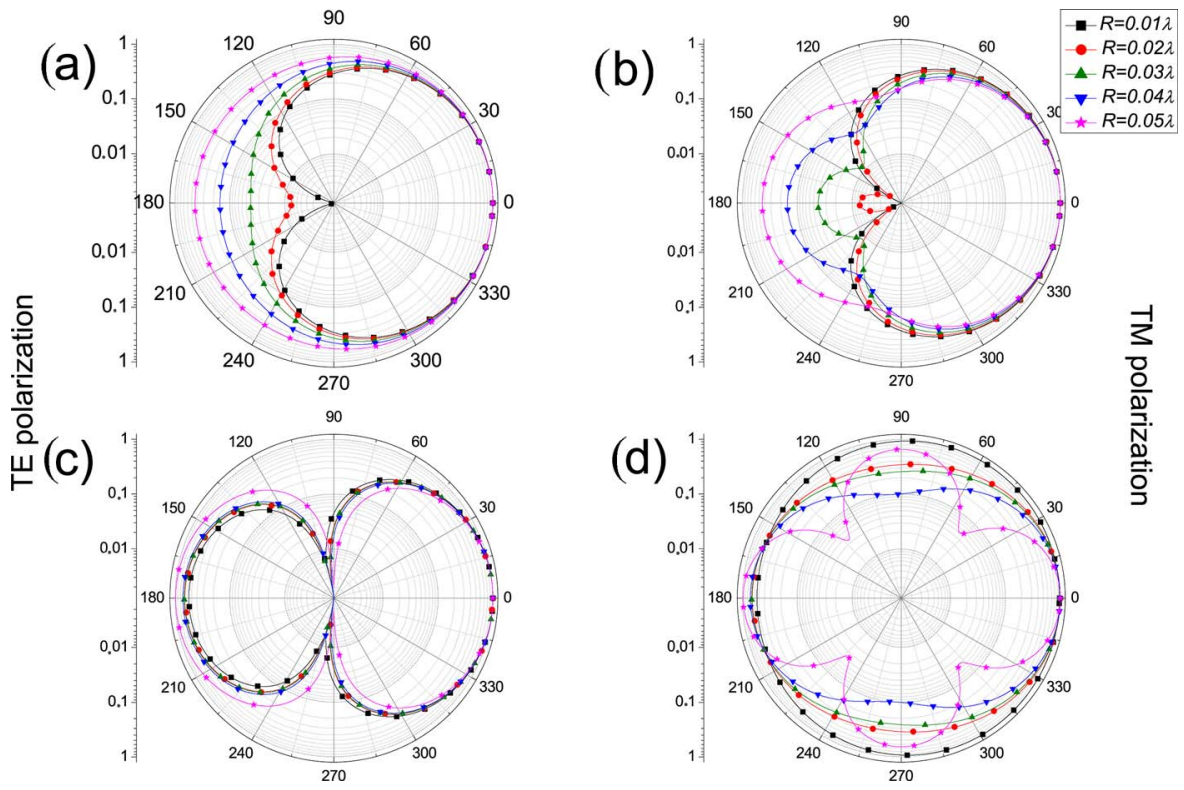

Fig. 7. (Color online) Normalized scattering diagrams when electric dipolar (a),(b) and electric quadrupolar (c),(d) resonance is excited for different particle size. (a), (c) Figures correspond to TE incident polarization; (b) (d) to TM polarization. 

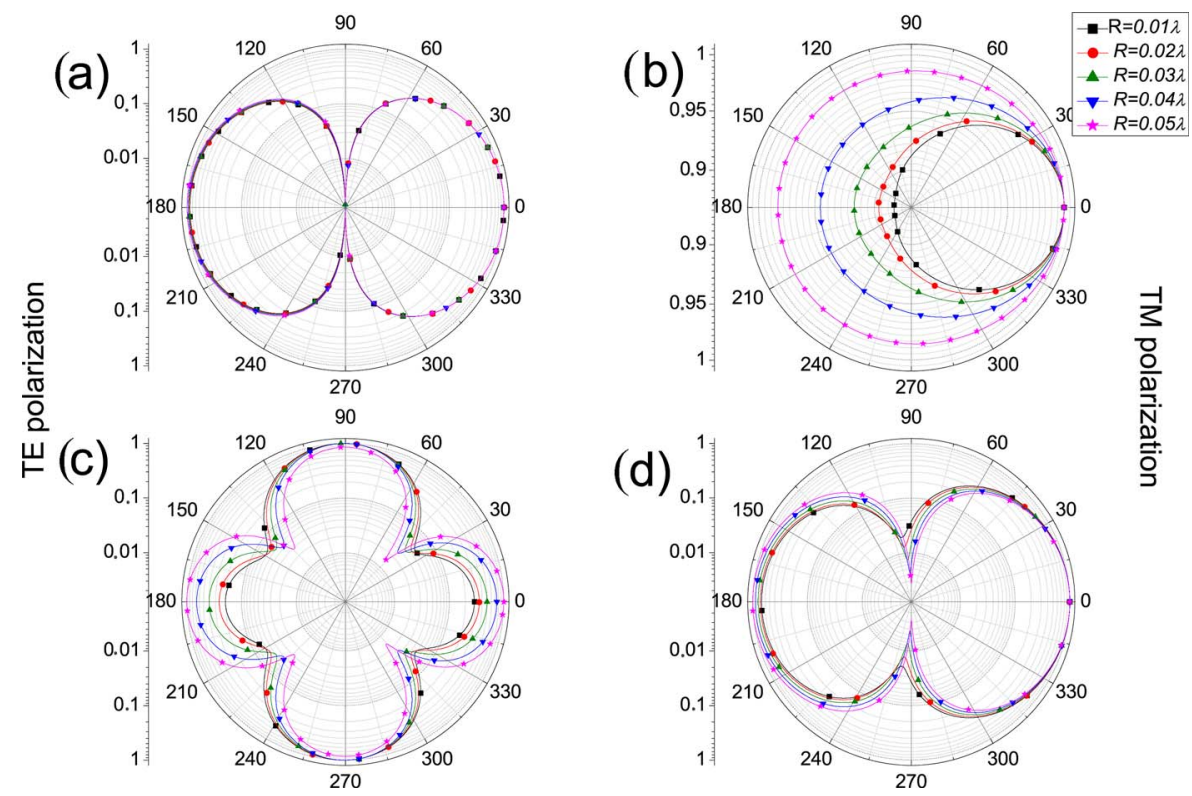

Fig. 8. (Color online) Normalized scattering diagrams when magnetic dipolar (a),(b) and magnetic quadrupolar (c),(d) resonance is excited for different particle size. (a), (c) Figures correspond to TE incident polarization; (b), (d) to TM polarization. The scale shown in (b) is for a better visualization of the curves.

Table 1. Maximum Intensity of Light Scattered by a Small Sphere as a Function of Size of the Sphere and for Both Polarizations of the Incident Light When an Electric Resonance Is Excited ${ }^{a}$

\begin{tabular}{cccccc}
\hline & \multicolumn{2}{c}{$\mathrm{TE}$} & & \multicolumn{2}{c}{$\mathrm{TM}$} \\
\cline { 2 - 3 } \cline { 5 - 6 }$R / \lambda$ & Dipolar & Quadrupolar & & Dipolar & Quadrupolar \\
\hline 0.01 & $0.05418\left(0^{\circ}\right)$ & $0.01234\left(0^{\circ}\right)$ & & $0.05418\left(0^{\circ}\right)$ & $0.01234\left(0^{\circ}\right)$ \\
0.02 & $0.06131\left(0^{\circ}\right)$ & $0.0187\left(0^{\circ}\right)$ & & $0.06131\left(0^{\circ}\right)$ & $0.0187\left(0^{\circ}\right)$ \\
0.03 & $0.07554\left(0^{\circ}\right)$ & $0.02087\left(0^{\circ}\right)$ & & $0.07554\left(0^{\circ}\right)$ & $0.02087\left(0^{\circ}\right)$ \\
0.04 & $0.09727\left(0^{\circ}\right)$ & $0.02088\left(0^{\circ}\right)$ & & $0.09727\left(0^{\circ}\right)$ & $0.02088\left(0^{\circ}\right)$ \\
0.05 & $0.09961\left(0^{\circ}\right)$ & $0.20629\left(0^{\circ}\right)$ & & $0.09961\left(0^{\circ}\right)$ & $0.20629\left(0^{\circ}\right)$ \\
\hline
\end{tabular}

${ }^{a}$ In parentheses, we include the angle at which the maximum appears.

ticular pair of values produces forward scattering different from zero. This must be considered an exception to the rule of zero forward established by Kerker.

\section{CONCLUSIONS}

We have proposed new generalized and approximate expressions for the first four Mie coefficients for spherical particles with electric and/or magnetic response whose size does not exceed $0.1 \lambda$.

Using these expressions we have studied light scattering resonances, dipolar and quadrupolar, for different optical properties, $\epsilon<0, \epsilon>0, \mu<0$, and $\mu>0$. Typical resonances due to coefficients $a_{1}, b_{1}$, and $b_{2}$ for $\epsilon>0$ and $\mu$ $=1$ [20] have been reproduced accurately. Furthermore, we have analyzed how these modes shift to lower values of $\epsilon$ as magnetic permeability increases from $\mu=1$.

Because of the recent developments with doublenegative materials $(\epsilon<0, \mu<0)$ in the optical range, we have paid special attention to the analysis of resonances in this range. It is remarkable the symmetry shown by the electric resonances that appear at $\epsilon \sim-2$ (dipolar) and
Table 2. Maximum Intensity of Light Scattered by a Small Sphere as a Function of Size of the Sphere and for both Polarizations of the Incident Light When a Magnetic Resonance Is Excited ${ }^{a}$

\begin{tabular}{lccllll}
\hline & \multicolumn{2}{c}{$\mathrm{TE}$} & & \multicolumn{2}{c}{$\mathrm{TM}$} \\
\cline { 2 - 3 } \cline { 5 - 7 }$R / \lambda$ & Dipolar & Quadrupolar & & Dipolar & \multicolumn{2}{c}{ Quadrupolar } \\
\hline 0.01 & $0.05077\left(0^{\circ}\right)$ & $0.04527\left(90^{\circ}\right)$ & $0.05321\left(0^{\circ}\right)$ & 0.01299 & $\left(0^{\circ}\right)$ \\
0.02 & $0.05248\left(0^{\circ}\right)$ & $0.05163\left(90^{\circ}\right)$ & & $0.05471\left(0^{\circ}\right)$ & 0.01799 & $\left(0^{\circ}\right)$ \\
0.03 & $0.05482\left(0^{\circ}\right)$ & $0.07001\left(270^{\circ}\right)$ & & $0.05665\left(0^{\circ}\right)$ & 0.0346 & $\left(0^{\circ}\right)$ \\
0.04 & $0.05689\left(0^{\circ}\right)$ & $0.11177\left(270^{\circ}\right)$ & & $0.05809\left(0^{\circ}\right)$ & 0.08278 & $\left(0^{\circ}\right)$ \\
0.05 & $0.05748\left(0^{\circ}\right)$ & $0.17536\left(180^{\circ}\right)$ & & $0.05819\left(0^{\circ}\right)$ & 0.1762 & $\left(180^{\circ}\right)$ \\
\hline
\end{tabular}

${ }^{a}$ In parentheses, we include the angle at which the maximum appears.

$\epsilon \sim-1.5$ (quadrupolar) and the magnetic ones that appear at $\mu \sim-2$ (dipolar) and $\mu \sim-1.5$ (quadrupolar). This symmetry tends to disappear as particle size increases.

In this work, we have observed that the position, shape, and number of resonances that appear depend strongly on the particle size. As particle radius increases, resonances shift and become less sharp and broader. These shifts depend on the type of the resonance; because of that we have studied these for the four modes due to $a_{1}, a_{2}, b_{1}$, and $b_{2}$. Furthermore, as expected, as particle size increases, more resonances appear.

In Section 4, we have studied scattering diagrams for different values of $\varepsilon$ and $\mu$ where representative resonances appear. In those we have shown the evolution of scattering as a function of particle size and how the influence of the dipolar and quadupolar terms changes. As $R$ increases, quadrupolar terms become to be important and scattering losses dipolar character. In addition, at $|\varepsilon|=|\mu|$ backscattering is zero [21].

In this study, we have used particle sizes in the range $R \sim 0.01 \lambda-0.1 \lambda$. For visible wavelengths, this is $R$ $\cong 10-100 \mathrm{~nm}$, so they are nanometric particles. 
The work presented simplified expressions for the first four Mie coefficients $\left(a_{1}, a_{1}, a_{2}, b_{1}, b_{2}\right)$ that permit determining the position and shape of the resonances without difficulty. This simplicity is a new step in the design of materials in the optical range with applications in fields like biomedicine, optics sensors, design of left-handed media devices, or optical storage of information.

\section{ACKNOWLEDGMENTS}

This research has been supported by Ministry of Education of Spain under projects FIS2004-06785 and FIS200760158. Braulio García-Cámara thanks the University of Cantabria for his Ph.D. grant.

\section{REFERENCES}

1. G. Mie, Ann. Phys. 25, 377 (1908).

2. L. Lorenz, Oeuvres Scientifiques (Johnson, 1964).

3. C. Bohren and D. Huffman, Absorption and Scattering of Light by Small Particles (Wiley, 1983).

4. M. I. Mishchenko, L. D. Travis, and A. A. Lacis, Scattering, Absorption and Emission of Light by Small Particles (Cambridge U. Press, 2002).

5. P. N. Prasad, Nanophotonics (Wiley-Interscience, 2004).

6. M. Kreuzer, R. Quidant, G. Badenes, and M. P. Marco, "Quantitative detection of doping substances by a localised surface plasmon sensor," Biosens. Bioelectron. 21, 1345-1349 (2006).

7. H. M. Hiep, T. Endo, K. Kerman, M. Chikae, D.-K. Kim, S. Yamamura, Y. Takamura, and E. Tamiya, "A localized surface plasmon resonance based immunosensor for the detection of casein in milk," Sci. Technol. Adv. Mater. 8, 331-338 (2007).

8. C. Guo, P. Boullanger, L. Jiang, and T. Liu, "Highly sensitive gold nanoparticle biosensor chips modified with a self-assembled bilayer for detection of Con A," Biosens. Bioelectron. 22, 1830-1834 (2007).

9. S. Pillai, K. R. Cathpole, T. Trupke, and M. A. Green, "Surface plasmon enhanced silicon solar cells," J. Appl. Phys. 101, 093105 (2007).

10. R. Ruppin, "Optical properties of small metal spheres," Phys. Rev. B 11, 2871-2876 (1975).

11. R. Ruppin, "Optical properties of a metal sphere with a diffuse surface," J. Opt. Soc. Am. 66, 449-453 (1976).

12. S. C. Hill and R. E. Benner, "Morphology-dependent resonances," in Optical Effects Associated with Small Particles, P. W. Barber and R. K. Chang, eds. (World Scientific, 1988).

13. G. W. Mulholland, C. F. Bohren, and K. A. Fuller, "Light scattering by agglomerates: coupled electric and magnetic dipole method," Langmuir 10, 2533-2546 (1994).

14. T. Jensen, L. Kelly, A. Lazarides, and G. Schatz,
"Electrodynamics of noble metal nanoparticles and nanoparticles clusters," J. Cluster Sci. 10, 295-317 (1999).

15. G. Roll and G. Schweiger, "Geometrical optics model of Mie resonances,” J. Opt. Soc. Am. A 17, 1301-1311 (2000).

16. B. M. Reinhard, M. Siu, H. Agarwal, A. P. Alivisatos, and J. Liphardt, "Calibration of a dynamic molecular ruler based on plasmon coupling between gold nanoparticles," Nano Lett. 5, 2246-2252 (2005).

17. F. Moreno, F. González, and J. M. Saiz, "Plasmon spectroscopy of metallic nanoparticles above flat dielectric substrates," Opt. Lett. 31, 1902-1904 (2006).

18. G. Lévêque and O. J. F. Martin, "Optical interactions in a plasmonic particle coupled to a metallic film," Opt. Express 14, 9971-9981 (2006).

19. O. Merchiers, F. Moreno, F. González, and J. M. Saiz, "Light scattering by an ensemble of interacting dipolar particles with both electric and magnetic polarizabilities," Phys. Rev. A 76, 043834 (2007).

20. G. Videen and W. S. Bickel, "Light scattering resonances in small spheres," Phys. Rev. A 45, 6008-6012 (1992).

21. M. Kerker, D. S. Wang, and L. Giles, "Electromagnetic scattering by magnetic spheres," J. Opt. Soc. Am. 73, $765-767$ (1983).

22. F. A. Pinheiro, A. S. Martinez, and L. C. Sampaio, "New effects in light scattering disordered media and coherent backscattering cone: Systems of magnetic particles," Phys. Rev. Lett. 84, 1435-1438 (2000).

23. O. Merchiers, F. Moreno, F. González, J. M. Saiz, and G. Videen, "Electromagnetic wave scattering from two interacting small spherical particles. Influence of their optical constants, $\varepsilon$ and $\mu$," Opt. Commun. 269, 1-7 (2007)

24. R. Ruppin, "Extinction properties of a sphere with negative permittivity and permeability," Solid State Commun. 116 411-415 (2000).

25. D. R. Smith, W. J. Padilla, D. C. Vier, S. C. Nemat-Nassser, and S. Schultz, "Composite medium with simultaneously negative permeability and permittivity," Phys. Rev. Lett. 84, 4184-4187 (2000).

26. V. Kuzmiak and A. A. Maradudin, "Scattering properties of a cylinder fabricated from a left-handed material," Phys. Rev. B 66, 045116 (2002).

27. R. Ruppin, "Surface polaritons and extinction properties of a left-handed material cylinder," J. Phys.: Condens. Matter 16, 5991-5998 (2004).

28. A. Alú, A. Salandrino, and N. Engheta, "Negative effective permeability and left-handed materials at optical frequencies," Opt. Express 14, 1557-1567 (2006).

29. C. M. Soukoulis, S. Linden, and M. Wegener, "Negative refractive index at optical wavelength," Science 315, 47-49 (2007).

30. V. Shalaev, "Optical negative-index metamaterials," Nat. Photonics 1, 41-48 (2007).

31. N. Engheta, "Circuits with light at nanoscales: Optical nanocircuits inspired by metamaterials," Science 317, 1698-1702 (2007). 